\title{
Advanced time domain wave-number sensing for structural acoustic systems. I. Theory and design
}

\author{
J. P. Maillard and C. R. Fuller \\ Vibration and Acoustics Laboratories, Mechanical Engineering Department, Virginia Polytechnic Institute \\ and State University, Blacksburg, Virginia 24061-0238
}

(Received 7 December 1992; revised 15 October 1993; accepted 26 January 1994)

\begin{abstract}
This paper discusses new work concerned with developing structural sensors and associated signal processing techniques that provide time domain estimates of far-field pressure or structural wave-number information. The sensor arrangement consists of multiple accelerometers whose outputs are passed through an array of linear filters. The impulse response of each filter is constructed from the appropriate Green's function for the elemental source area associated with each sensor. The outputs of the filter array are then summed in order to predict far-field pressure or wave-number information somewhat analogous to the well-known boundary element technique. A major significance of the approach is that it provides time domain information and can thus be efficiently applied to active structural acoustic control approaches.
\end{abstract}

PACS numbers: 43.40.At, 43.40.Rj, 43.60.Gk

\section{INTRODUCTION}

Structure-borne sound is an important problem in many applications, and it is of prime interest to predict acoustic radiation from vibrating structures. Indeed, the radiation information is often used to develop appropriate control approaches. Acoustic holography ${ }^{1}$ has been successfully applied to prediction of far-field radiation from real structures. For example, the technique may involve measurements of the acoustic pressure on a 2-D plane located in the near field using an array of microphones. The measured data are then transformed in the frequency and spatial domains to provide far-field information in a 3-D space. Structural acoustic systems have also been modeled using the boundary element method ${ }^{2}$. In this approach, the structural response, known at discrete locations, is used to construct a solution of the Helmoltz integral by solving a linear system. Both of the above methods allow the study of the radiation behavior of complex structures. However, the use of both methods results in extensive computations (FFT, matrix inversions, etc.) on a large amount of data. Therefore, "real time" prediction cannot be readily achieved. Here, we define "real time" to be in the time domain.

This paper, the first of two companion papers, presents a real time prediction technique using structural sensors. The analytical development will emphasize important aspects for application to the active structural acoustic control (ASAC) technique. ${ }^{3,4}$ In this approach, a feedforward adaptive controller minimizes one or several real time error signals representing acoustic radiation information. Traditionally, the error signals are the output of microphones located in the far field. However, the use of microphones is often impractical in real applications and research is now addressing the development of structural sensors to replace error microphones. In that respect, much attention has been focused on new sensing materials (PVDF films, optic fibers) originally used in active vibration control. ${ }^{3}$ When applied to acoustic radiation, the sensor should provide information related to the far-field pressure such that only the structural response contributing to radiation is observed. As a result, the controller dimension is reduced and better performances are achieved. Clark and Fuller ${ }^{4}$ have discussed the use of PVDF film modal sensors in the feedforward control approach applied to rectangular acoustic radiators. By choosing the appropriate location and shape of the PVDF film, only those structural modes that efficiently radiate to the far field are observed. In related work, Baumann et al. ${ }^{5}$ have developed radiation filters for use in state feedback approaches to map structural states to radiation states of the system and demonstrated their use in sound radiation control from impulsively excited structures.

In both of the above works, the sensor output provides global radiation information related to the total acoustic power radiated by the structure. The present work is concerned with estimating far-field pressure radiated in prescribed directions. The approach implements point structural sensors (i.e., accelerometers) in parallel with an array of digital filters to obtain an error signal directly related to the far-field pressure in a prescribed direction. Namely, the sensor output is shown to be proportional to a time-shifted version of the far-field radiated pressure. The present analysis is limited to estimation of radiation from planar surfaces of finite extent. The relation between structural response and far-field pressure becomes more complex in case of nonplanar geometries and will be investigated in future work.

In the first part, the theory underlying the technique is presented. Following the ideas developed in boundary element method, the Rayleigh's integral that gives the farfield pressure radiated from a planar source, is approximated by a finite summation of filtered acceleration signals measured at a number of points on the structure. Two related sensing approaches are analytically outlined. In the first configuration, the filter array is designed to model the 
transfer functions between the acceleration and the radiated pressure of the elemental source area associated with each sensor. Then, the same approach is applied to evaluate the acceleration wave-number component in a prescribed direction. The corresponding discrete summation can be referred to as the spatial discrete wave-number transform analogous to the well-known time domain discrete Fourier transform. It is shown that this wave-number domain error information yields the same control performances as the far-field pressure when used in a feedforward control approach. The second part of the paper discusses the practical implementation of the technique. Two design methods are proposed for constructing the digital filters. The first involves frequency domain design algorithms using the analytical transfer functions derived in the first part. The second approach implements time domain optimal filtering techniques that allow the filters to be designed from experimental data.

Results of computer simulations on the sensor design and its application to active control of radiation from a baffled planar radiator excited by broadband disturbances will be discussed in the companion paper in order to illustrate the use of the sensing procedure.

\section{THEORY}

\section{A. Pressure sensing configuration}

Based on Rayleigh's integral, an expression that depends solely on the structural acceleration measured at discrete points on the vibrating surface is derived for the far-field pressure radiated from planar sources.

\section{Rayleigh's integral}

The Helmoltz integral equation relates the pressure field complex amplitude $P(\mathbf{r})$ at field point $\mathbf{r}$ in terms of the surface pressure $P\left(r_{0}\right)$ and its gradient defined over the radiating surface $S\left(\mathrm{r}_{0} \in S\right)$. Assuming a harmonic time dependence of the form $e^{j \omega t}$, where $\omega$ is the angular frequency of the source, the Helmoltz integral can be written as ${ }^{6}$

$$
\begin{aligned}
P(\mathbf{r})= & \int_{S}\left(P\left(\mathbf{r}_{0}\right) \frac{\partial g}{\partial \eta}\left(\mathbf{r}-\mathbf{r}_{0}\right)\right. \\
& \left.-g\left(\mathbf{r}-\mathbf{r}_{0}\right) \frac{\partial P}{\partial \eta}\left(\mathbf{r}_{0}\right)\right) d S \quad(\mathbf{r} \notin S),
\end{aligned}
$$

where $\eta$ is the outward normal unit vector and $g(\mathbf{r})$, the free-space Green's function defined in the free field as $g(\mathbf{r})=(1 / 4 \pi|\mathbf{r}|) \exp \left(j k_{0}|\mathbf{r}|\right) ; k_{0}=\omega / c$ is the acoustic wave number, and $c$ is the speed of sound. Applying the boundary condition prescribed over $S$,

$$
\frac{\partial P}{\partial \eta}\left(\mathrm{r}_{0}\right)=-\rho \ddot{W}\left(\mathrm{r}_{0}\right) \quad\left(\mathrm{r}_{0} \in S\right),
$$

where $\rho$ is the fluid density, the pressure field is expressed in terms of the surface pressure and the fluid particle acceleration over the radiating surface, the latter being equal to the structural normal acceleration distribution $\ddot{W}\left(\mathbf{r}_{0}\right)=-\omega^{2} W\left(\mathbf{r}_{0}\right)$. In the above notation, $\ddot{W}\left(\mathbf{r}_{0}\right)$ refers to the complex amplitude of the second time derivative of the normal displacement $w\left(\mathbf{r}_{0}, t\right)=W\left(\mathbf{r}_{0}\right) e^{j \omega t}$.

In the case of an infinite planar source, it can be shown that the surface pressure term vanishes when replacing the free-space Green's function $g(r)$ by $G(r)=2 g(r)$, which satisfies the Neumann boundary condition $\partial G / \partial \eta(r)=0$ for $r \in S$,

$$
P(\mathrm{r})=2 \rho \int_{S} g\left(\mathrm{r}-\mathrm{r}_{0}\right) \ddot{W}\left(\mathrm{r}_{0}\right) d S\left(\mathrm{r}_{0}\right) .
$$

When $\ddot{W}\left(r_{0}\right)$ is nonzero over a finite surface (i.e., the structure is finite and located in an infinite baffle), analytical evaluation of the above representation becomes possible only in the far field. Removing the denominator of the Green's function from the integral produces the Rayleigh's formula for planar radiators ${ }^{6}$

$$
P(\mathbf{r})=\frac{\rho}{2 \pi r} \int_{S} e^{-j k_{0}\left|\mathbf{r}-\mathbf{r}_{0}\right|} \ddot{W}\left(\mathbf{r}_{0}\right) d S\left(\mathbf{r}_{0}\right) \quad\left(|\mathbf{r}| \gg\left|\mathbf{r}_{0}\right|\right),
$$

where $r=|\mathbf{r}|$. This formulation can be physically interpreted as an infinite sum of monopoles with volume acceleration $\ddot{W}\left(\mathrm{r}_{0}\right) d S\left(\mathrm{r}_{0}\right)$ located on an infinite rigid planar surface (in this case the amplitude of the free point source is multiplied by a factor of 2 ).

\section{Discrete Rayleigh's integral}

In practice, the structural response can only be evaluated at a finite number of points by means of point transducers such as accelerometers. Hence, it is necessary to approximate the far-field pressure, expressed in Eq. (4) with continuous coordinates, by an expression involving a finite number, $N_{d}$, of discrete coordinates $\left(\mathbf{r}_{i} \in S_{0}\right.$, $\left.i=1,2, \ldots, N_{d}\right)$. The resulting approximation will be referred to as the discrete Rayleigh's integral. Following the same idea developed in the boundary element method, the surface integral is approximated by a discrete summation over the integrand evaluated at a finite number of points. The surface $S$ is discretized in $N_{d}$ surfaces $S_{i}\left(S=S_{1} \cup S_{2}\right.$ $\cup \cdots \cup S_{N_{d}}$ ) such that a piecewise constant approximation for $\dot{W}\left(\mathrm{r}_{0}\right)$ can be made over each $S_{i}$, i.e., $\ddot{W}\left(r_{0}\right) \simeq \ddot{W}\left(r_{i}\right)$ on $S_{i}$. Substituting $\ddot{W}\left(r_{0}\right)$ in Eq. (4), the radiated pressure can then be approximated by

$$
P(\mathbf{r}) \simeq \frac{\rho}{2 \pi r} \sum_{i=1}^{N_{d}} \ddot{W}\left(\mathbf{r}_{i}\right) \int_{S_{i}} e^{-j k_{0}\left|\mathbf{r}-\mathbf{r}_{0}\right|} d S_{i}\left(\mathbf{r}_{0}\right) .
$$

The above expression defines the piston approximation: the far-field pressure is constructed by summing the radiated pressure of $N_{d}$ pistons with uniform acceleration distribution $\ddot{W}\left(\mathbf{r}_{i}\right)$ and surface $S_{i}$. Assuming $\exp \left(-j k_{0}\left|\mathbf{r}-\mathbf{r}_{0}\right|\right)$ is almost constant over each elemental surface $S_{i}$, a simplified expression is obtained by removing the exponential term from the integral in Eq. (5),

$$
P(\mathbf{r}) \simeq \frac{\rho}{2 \pi r} \sum_{i=1}^{N_{d}} \ddot{W}\left(\mathbf{r}_{i}\right) e^{-j k_{0}\left|\mathbf{r}-\mathbf{r}_{i}\right|} S_{i} .
$$

The far-field pressure is now approximated by the acoustic radiation of $N_{d}$ monopole sources of volume acceleration $\ddot{W}\left(\mathbf{r}_{i}\right) S_{i}$ located on a rigid baffle. Equation (6) is referred 
to as the monopole approximation. Both approximations are equivalent in the far field when the acoustic wavelength is much larger than the characteristic dimension $a_{i}$ of the elemental surfaces $S_{i}\left(k_{0} a_{i}<1\right)$, i.e., in the far field, the rigid piston can be replaced by a monopole source. As the radiation control will be applied in the low-frequency range, the monopole approximation is used here.

\section{Sensing approach}

Introducing the time dependance, $\ddot{w}\left(\mathbf{r}_{i}, t\right)=\ddot{W}\left(\mathbf{r}_{i}\right) e^{j \omega t}$, in Eq. (6), the discrete far-field pressure is expressed as the summation of the filtered acceleration signals measured at locations $\mathbf{r}_{i}$ on the structure,

$$
p_{d}(\mathbf{r}, t)=P_{d}(\mathbf{r}) e^{j \omega t}=\sum_{i=1}^{N_{d}} H_{i}(\omega) \ddot{W}\left(\mathbf{r}_{i}\right) e^{j \omega t} .
$$

The frequency response functions $H_{i}(\omega)$ are written substituting $k_{0}=\omega / c$ in Eq. (6) as

$$
H_{i}(\omega)=\frac{\rho S_{i}}{2 \pi r} \exp \left(-j \omega \frac{\left|\mathbf{r}-\mathbf{r}_{i}\right|}{c}\right), \quad i=1,2, \ldots, N_{d} .
$$

The dependence of $H_{i}(\omega)$ on the field point $\mathbf{r}$ has been omitted for brevity. It is seen from Eq. (8) that the frequency response functions to be modeled present a constant magnitude and a linear phase with a positive acoustic time delay $\tau_{i}=\left|\mathbf{r}-\mathbf{r}_{i}\right| / c$ making the frequency response functions causal. This important property motivates the use of finite impulse response filters in the sensor implementation.

Now, the sensor is described in the discrete time domain. The signals are sampled with the sampling frequency $F_{s}$. FIR filters are commonly represented in terms of their discrete impulse response $\left\{U_{l}, l=0,1, \ldots, L\right\}$. In matrix form, the output $y(n)$ of the filter at time $t_{n}$ is expressed as

$$
y(n)=\mathbf{U}^{T} \mathbf{X}(n)=\sum_{l=0}^{L} U_{p} x(n-l),
$$

where $\mathbf{U}$ is a column vector containing the filter coeffcients,

$$
\mathrm{U}=\left[\begin{array}{llll}
U_{0} & U_{1} & \cdots & U_{L}
\end{array}\right]^{T}
$$

and $\mathbf{X}(n)$ is a column vector containing the filter input sequence,

$$
\mathbf{X}=\left[\begin{array}{llll}
x(n) & x(n-1) & \cdots & x(n-L)
\end{array}\right]^{T} .
$$

Here, $T$ denotes the transpose operator. Using the above representation, the sensor output $e(n)$ at time $t_{n}$ is expressed as

$$
e(n)=\sum_{i=1}^{N_{d}} \mathbf{U}^{i T} \mathbf{X}^{i}(n),
$$

where $\mathrm{X}^{i}(n)$ is the $i$ th sampled acceleration signal at time $t_{n}$ and $\mathrm{U}^{i}$ the impulse response of the associated FIR filter for the ith accelerometer. The above configuration can be extended to a multiple output sensor involving a set of filter arrays. The $j$ th output becomes

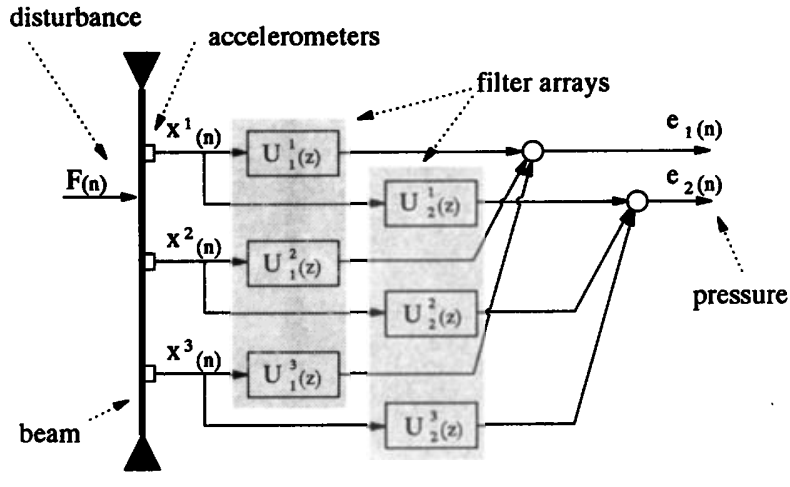

FIG. 1. Sensing configuration applied to a 1-D beam.

$$
e_{j}(n)=\sum_{i=1}^{N_{d}} \mathbf{U}_{j}^{i T} \mathbf{X}^{i}(n)
$$

where $\mathbf{U}_{j}^{i}(n)$ denotes the $i$ th impulse response of the $j$ th filter array. As an example, Fig. 1 presents the block diagram of the sensing configuration applied to a baffled simply supported beam. In this arrangement, two filter arrays processing the output of three accelerometers give the pressure at two different locations in the far field.

\section{B. Wave-number component sensing configuration}

The same approach is now applied to the evaluation of the structural wave-number component. This second sensing configuration is shown to be equivalent to the first method in terms of radiated energy and thus, can be used in a feedforward control approach. Moreover, using the analogy with the time domain discrete Fourier transform, the wave-number transform approach allows for an investigation of spatial sampling and resultant aliasing.

\section{Wave-number transform of a 2-D rectangular radiator}

Using rectangular coordinates, the spatial response of a 2-D planar source is represented in the wave-number domain in terms of its acceleration distribution $\ddot{W}(x, y)$ and the components $k_{x}$ and $k_{y}$ of the structural wave number $\mathbf{k}_{f}$ in the $x$ and $y$ direction, respectively,

$$
\tilde{\ddot{W}}\left(k_{x}, k_{y}\right)=\int_{-\infty}^{+\infty} \int_{-\infty}^{+\infty} \ddot{W}(x, y) e^{j\left(k_{x} x+k_{y} y\right)} d x d y .
$$

The time dependence $e^{j \omega t}$ has been omitted for brevity. The above surface integral can be interpreted as the spatial Fourier transform of the acceleration distribution $\ddot{W}(x, y)$. In the case of a finite rectangular radiator with length $L_{x}$ and width $L_{y}$ located in an infinite rigid baffle, Eq. (14) simplifies to

$$
\tilde{\ddot{W}}\left(k_{x}, k_{y}\right)=\int_{-L_{x} / 2}^{+L_{x} / 2} \int_{-L_{y} / 2}^{+L_{y} / 2} \ddot{W}(x, y) e^{j\left(k_{x} x+k_{y} y\right)} d x d y .
$$

The above wave-number domain representation allows the study of the acoustic energy radiated in the far field directly from the structural response. As shown in Ref. 7, 


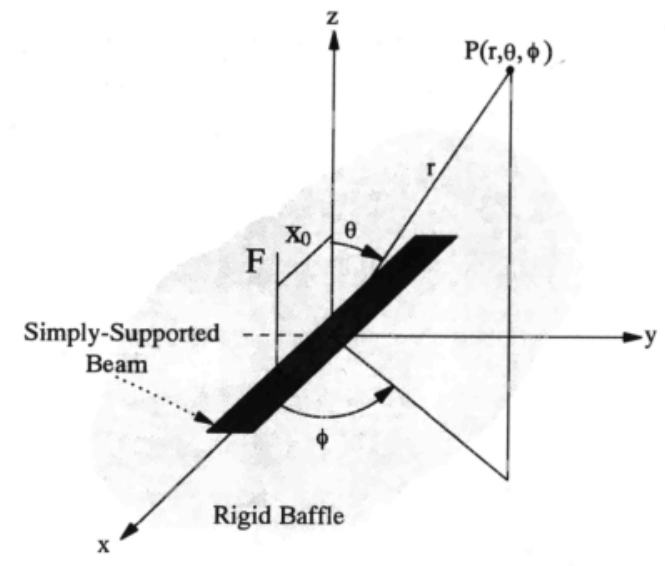

FIG. 2. Coordinate system of the simply supported beam.

the far-field radiated energy is due only to the supersonic wave numbers, $\mathbf{k}_{f}$, smaller than the acoustic wave number $k_{0}=\omega / c$,

$$
\left(k_{x}^{2}+k_{y}^{2}\right)^{1 / 2} \leqslant k_{0} .
$$

It follows that a wave-number sensor designed for acoustic radiation control will be required to observe only low values of wave numbers. ${ }^{8}$

The relation between the wave-number information and the acoustic radiated pressure is now outlined. The Rayleigh's integral is written in the spherical coordinates $(r, \theta, \phi)$ defined in Fig. 2 using the following approximation:

$$
\left|\mathbf{r}-\mathbf{r}_{0}\right| \simeq r-x_{0} \sin \theta \cos \phi-y_{0} \sin \theta \sin \phi .
$$

Substituting Eq. (17) in Eq. (4) yields

$$
\begin{aligned}
P(r, \theta, \phi)= & \frac{\rho e^{-j k_{0} r}}{2 \pi r} \int_{-L_{x} / 2}^{L_{x} / 2} \int_{-L_{y} / 2}^{L_{y} / 2} \ddot{W}\left(x_{0}, y_{0}\right) \\
& \times e^{j k_{0} \sin \theta\left(x_{0} \cos \phi+y_{0} \sin \phi\right)} d x_{0} d y_{0} .
\end{aligned}
$$

By comparing Eqs. (15) and (18), the far-field pressure can now be expressed in terms of structural information as

$$
p(r, \theta, \phi, t)=\frac{\rho e^{-j k_{0} r}}{2 \pi r} \tilde{\ddot{W}}\left(k_{x}, k_{y}\right) e^{j \omega t},
$$

where $k_{x}$ and $k_{y}$ are defined by

$$
k_{x}=k_{0} \sin \theta \cos \phi, \quad k_{y}=k_{0} \sin \theta \sin \phi .
$$

Multiplying both sides of Eq. (19) by the complex conjugate quantities yields

$$
|P(r, \theta, \phi)|^{2}=(\rho / 2 \pi r)^{2}\left|\tilde{\ddot{W}}\left(k_{x}, k_{y}\right)\right|^{2} .
$$

From Eq. (21), it is seen that the acoustic energy radiated in the direction defined by $\theta$ and $\phi$ is determined solely by the structural wave-number component corresponding to $k_{x}$ and $k_{y}$ in Eq. (20). These values of $k_{x}$ and $k_{y}$ are always smaller than the acoustic wave number $k_{0}=\omega / c$; they correspond to the supersonic structural wave numbers that characterize the radiating components of the structural vibration. Therefore, a sensor that evaluates the wavenumber component at prescribed values of $k_{x}$ and $k_{y}$ de- fined in Eq. (20) can effectively replace an error microphone located in the far field at angles $\theta$ and $\phi$. Such a sensor will be referred to as a structural wave-number domain sensor.

\section{Discrete wave-number transform}

As done previously, the integral in Eq. (15) is approximated using $N_{d}$ rectangular elements of dimension $\Delta x$ by $\Delta y$ by a finite summation in the form

$$
\tilde{W}\left(k_{x}, k_{y}\right) \simeq \Delta x \Delta y \sum_{n_{x}=1}^{N_{x}} \sum_{n_{y}=1}^{N_{y}} \ddot{W}\left(x_{n_{x}}, y_{n_{y}}\right) e^{j\left(k_{x} x_{n_{x}}+k_{y} y_{n_{y}}\right)},
$$

where $\Delta x=L_{x} / N_{x}$ and $\Delta y=L_{y} / N_{y}\left(N_{x}\right.$ and $N_{y}$ are the number of elements in the $x$ and the $y$ direction, respectively). The collocation points $\left(x_{n_{x}}, y_{n_{y}}\right)$ are defined by $x_{n_{x}}=-L_{x} / 2+\Delta x / 2\left(2 n_{x}-1\right)$ and $y_{n_{y}}=-L_{y} / 2+\Delta y /$ $2\left(2 n_{y}-1\right)$, where $n_{x}=1,2, \ldots, N_{x}$ and $n_{y}=1,2, \ldots, N_{y}$.

Introducing the time dependence, the discrete wavenumber component is expressed as the summation of the acceleration signals $\ddot{w}\left(x_{n_{x}}, y_{n_{y}}, t\right)=\ddot{W}\left(x_{n_{x}}, y_{n_{y}}\right) e^{j \omega t}$ filtered by the frequency response function $H_{n_{x} n_{y}}(\omega)$,

$$
\begin{aligned}
\tilde{\ddot{w}}_{d}\left(k_{x}, k_{y}, t\right) & =\tilde{\ddot{W}}_{d}\left(k_{x}, k_{y}\right) e^{j \omega t} \\
& =\sum_{n_{x}=1}^{N_{x}} \sum_{n_{y}=1}^{N_{y}} H_{n_{x} n_{y}}(\omega) \ddot{W}\left(x_{n_{x}}, y_{n_{y}}\right) e^{j \omega t},
\end{aligned}
$$

where

$$
H_{n_{x} n_{y}}(\omega)=\Delta x \Delta y \exp \left(j \omega \tau_{n_{x^{n_{y}}}}\right) .
$$

The above frequency response functions present a constant time delay,

$$
\tau_{n_{x^{n}}}=\frac{x_{n_{x}} \sin \theta \cos \phi+y_{n_{y}} \sin \theta \sin \phi}{c} .
$$

Recalling the time convention $e^{j \omega t}$, the frequency response functions in Eq. (24) can be modeled by causal, linear phase FIR filters only if the time delay defined in Eq. (25) is negative. Since $\tau_{n_{x} n_{y}}$ take both positive and negative values, a modified delay must be introduced in order to make the filters realizable.

It is next demonstrated that the above requirement that results in a time-shifted error information can be satisfied in feedforward control. In this case, the controller is defined so as to minimize the mean square value of the sensor output, i.e., $J=E\left\{e^{2}(t)\right\}$ (Ref. 9). After control (after convergence in the case of an adaptive structure), the error signal $e(t)$ can be assumed to be stationary in a steady-state disturbance, i.e., $E\left\{e^{2}(t)\right\}=E\left\{e^{2}(t-\Delta t)\right\}$, where $\Delta t$ denotes a time delay. This important property implies that a time-shifted version of the actual error signal will result in the same control performances. Also, it is straightforward to show that the error signal $e(t)$ is of arbitrary magnitude: since the optimal solution for the controller is found by setting the partial derivatives of $J$ with 
respect to the compensator coefficients to zero, any constant multiplicative factor will cancel. Note that the above result does not apply to model reference control; ${ }^{10}$ in that case, the error signal is required to match a prescribed reference signal instead of being minimized. Moreover, when using several error signals, the proportionality constant should be the same for each sensor array. The above results can be verified by plotting the cost functions $J=E\left\{e^{2}(t)\right\}$ and $\bar{J}=E\left\{\bar{e}^{2}(t)\right\}$ versus the weights of the compensator where $\bar{e}(t)=\alpha e(t-\Delta t)$ is a proportional time-shifted version of the original error signal ( $\alpha$ constant factor). It is seen that both surfaces have the same minimum but different shapes. Hence, a proportional timeshifted error signal influences the convergence process of the feedforward adaptive controller but does not alter the optimal solution.

The above discussion yields several important results. First, in order to satisfy the causality requirements of the frequency responses in Eq. (24), the time delays $\tau_{n_{x} n_{y}}$ can be replaced by $\bar{\tau}_{n_{x} n_{y}}=\tau_{n_{x} n_{y}}-\Delta t$, where $\Delta t>0$ is such that each modified time delay $\bar{\tau}_{n_{x} n_{y}}$ be negative. Second, the equivalence between the pressure and wave-number information can be further analyzed. Recalling Eq. (19), the wave-number component evaluated at $k_{x}$ and $k_{y}$ given by Eq. (20) appears to be proportional to the time-shifted acoustic pressure $p(r, \theta, \phi, t+r / c)$ radiated in the direction defined by $\theta$ and $\phi$. It follows that using the wave-number information as an error signal in a feedforward control approach leads the same optimal solution as the far-field pressure information. This gives a physical interpretation of the wave-number component evaluated in the supersonic region and confirms the validity of its use as error information.

\section{Wave-number spectrum}

The previous section derived the transfer functions used to evaluate a single wave-number component $\ddot{W}_{d}\left(k_{x}, k_{y}\right)$. The approach is now formally extended to provide information over the entire wave-number spectrum.

As shown previously, only the supersonic wave numbers are of interest in radiation control, i.e., $\left(k_{x}^{2}\right.$ $\left.+k_{y}^{2}\right)^{1 / 2} \leqslant k_{0}$. Physically, they relate to a particular direction of radiation at angles $\theta$ and $\phi$. Hence, a multiple output structural sensor is built by choosing discrete values of $k_{x}, k_{y}$ and constructing for each of them the appropriate transfer functions as given in Eq. (24). In order to obtain a constant time delay over the frequency bandwidth (linear phase), $k_{x}$ and $k_{y}$ must also satisfy Eq. (20) where $\theta$ and $\phi$ are fixed. In other words, the structural wave numbers must be proportional to the driving frequency.

The diagram of Fig. 3 shows the discrete wave-number spectrum for a 1-D structure $\left(k_{y}=0\right)$ at two driving frequencies $\omega_{1}$ and $\omega_{2}$. The solid lines represent the modulus of the discrete wave-number component defined in Eq. (23). The dots represent the same quantity evaluated by a multiple output sensor at three discrete values of $k_{x}$ between $-k_{0}$ and $k_{0}$. Each of these discrete values corre-

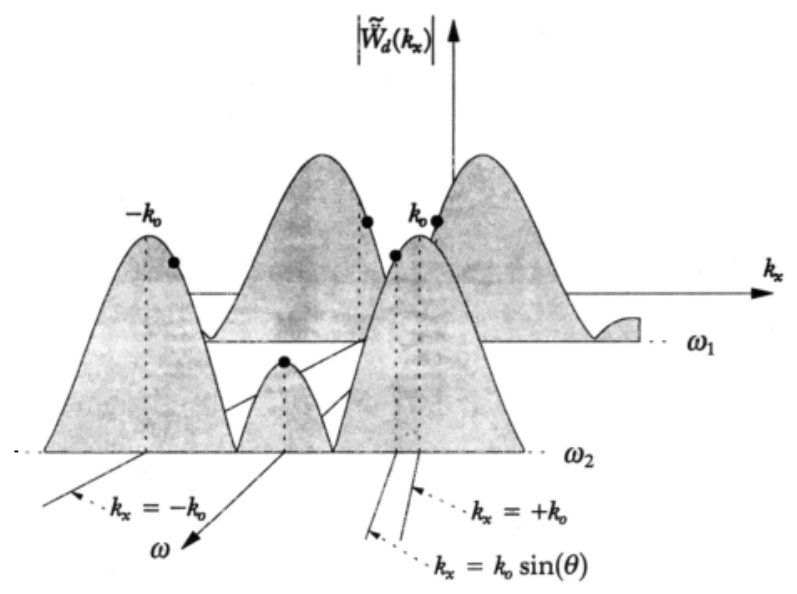

FIG. 3. Typical multiple output wave-number sensor diagram.

spond to a fixed direction of radiation $(\theta, \phi=0)$ and thus, are located on lines of equation $k_{x}=\omega / c \sin \theta$ in the $\left(k_{x}, \omega\right)$ plane. Therefore, the frequency response of the sensor output in the direction $\theta$ is obtained by plotting the wave-number component corresponding to $k_{x}$ located on this line.

As an example, Fig. 4 presents the magnitude and phase of the continuous [Eq. (15)] and discrete [Eq. (23)] wave-number components evaluated along the direction $(\theta$ $=75^{\circ}, \phi=0$ ) versus frequency for a simply supported beam. The phase angle is relative to the input disturbance. The closed-form expressions for the continuous and discrete wave-number components are derived in the appendix. The beam characteristics and natural frequencies are given in Tables I and II, respectively. The model assumed $0.15 \%$ damping in the 20 modes included in the beam analytical response [Eqs. (A1) and (A3): $\eta_{p}=0.0015$,

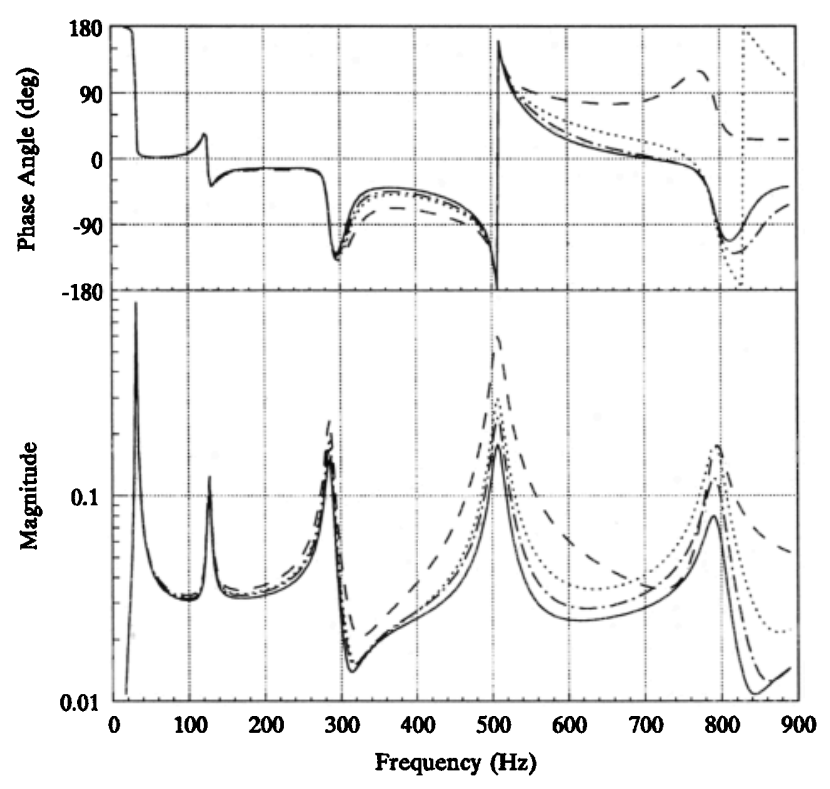

FIG. 4. Wave-number component corresponding to $\theta=75^{\circ}$ for a simply supported beam; (a) ——, continuous representation; (b) -- -, (c) ..-., (d) ........, discrete representations using three, four, and five point sensors, respectively. 
TABLE I. Beam characteristics.

\begin{tabular}{lc}
\hline \hline Length & $0.38 \mathrm{~m}$ \\
Width & $0.04 \mathrm{~m}$ \\
Section & $0.0002 \mathrm{~m}^{2}$ \\
Mass density & $3132 \mathrm{~kg} / \mathrm{m}^{3}$ \\
Bending stiffness & $5.3290 \mathrm{~N} / \mathrm{m}^{2}$ \\
\hline \hline
\end{tabular}

$p=1,2, \ldots, 20]$. The structure is surrounded by air with density $\rho=1.27 \mathrm{~kg} / \mathrm{m}^{3}$ and sound velocity $c=343 \mathrm{~m} / \mathrm{s}$. The disturbance point force has magnitude $F=0.35 \mathrm{~N}$ and is located at $x_{0}=-0.15 \times L_{x} / 2$. Three, four, and five accelerometers are used respectively to provide wave-number information over the first five structural modes. As expected, the overall error between continuous and discrete representations decreases when the number of accelerometers is increased. In the three cases [curves (b), (c), and (d)], negligible error is noticed for frequencies up to the third mode. The error becomes significant above the third mode when a three point sensor is used. However, four and five point sensors give good approximation up to the fifth mode, the best result being obtained with $N_{d}=5$. To summarize, the far-field radiation of the low order structural modes of a simply supported beam is roughly approximated by a small number of monopole sources equally spaced on the beam. Note that other types and locations of excitation would show similar behaviors. The next section discusses how these errors are related to the discrete approximation. Their influence on the control performances will be addressed in the companion paper.

\section{Analogy of the discrete wave-number transform with the time domain discrete Fourier transform-Sampling aspects}

Analogous to the time domain Fourier transform and its discrete representation (DFT), the discretization mechanism of the wave-number sensing procedure is further investigated. The case of the simply supported beam used previously is considered here for simplicity.

Recalling Eqs. (15) and (23), the continuous and discrete wave-number components become, respectively,

$$
\begin{aligned}
& \tilde{\ddot{W}}\left(k_{x}\right)=L_{y} \int_{-L_{x} / 2}^{+L_{x} / 2} \ddot{W}(x) e^{j k_{x} x} d x, \\
& \widetilde{\mathscr{W}}_{d}\left(k_{x}\right)=L_{y} \Delta x \sum_{i=1}^{N_{d}} \ddot{W}\left(x_{i}\right) e^{j k_{x} x_{i}},
\end{aligned}
$$

where $x_{i}=-L_{x} / 2+\Delta x / 2(2 i-1), i=1,2, \ldots, N_{d}$. The above representations are analogous to the time domain Fourier

TABLE II. Beam analytical natural frequencies.

\begin{tabular}{cc}
\hline \hline Mode & Natural frequency $(\mathrm{Hz})$ \\
\hline 1 & 31.73 \\
2 & 126.9 \\
3 & 285.6 \\
4 & 507.7 \\
5 & 793.2 \\
6 & 1142 \\
\hline \hline
\end{tabular}

transform. The time variable is replaced by the spatial coordinate $x, \Delta x$ being the sampling period, and the frequency variable by the structural wave number or spatial frequency, $k_{x}$. The beam response, $\ddot{W}(x)$, can thus be seen by analogy as a time domain signal of finite duration corresponding to the length $L_{x}$ and its discretized version, $\ddot{W}\left(x_{i}\right), i=1,2, \ldots, N_{d}$, as a $N_{d}$ sample sequence with sampling period corresponding to $\Delta x$, where $\Delta x=L_{x} / N_{d}$.

Applying the properties of the time domain DFT, a fundamental relation between continuous and discrete representations in the wave-number domain is expressed as

$$
\widetilde{\ddot{W}}_{d}\left(k_{x}\right)=\tilde{\ddot{W}}\left(k_{x}\right) * \delta_{N}\left(k_{x}\right),
$$

where $*$ denotes the convolution product. The spectral window $\delta_{N}\left(k_{x}\right)$ solely depends on the sensor arrangement, i.e., location and number of point sensors. In the case of equal spacing, it takes the closed form given by Eq. (A16) of the Appendix. The representation in Eq. (28) is useful since the influence of the sensor arrangement on the wavenumber component estimate is solely determined by $\delta_{N}\left(k_{x}\right)$. As described in the Appendix, the spectral window obtained in the case of equally spaced point sensors has large amplitudes at wave numbers $k_{x}=n K_{s}$, where $K_{s}=2 \pi / \Delta x$ is the spatial sampling frequency and $n$ is an integer. It follows that significant contribution to the discrete wave-number component $\ddot{W}_{d}\left(k_{x}\right)$ comes from continuous wave-number components that are far removed from the wave numbers of interest, i.e., $\ddot{W}\left(k_{x}-n K_{s}\right)$. Unless the continuous wave-number spectrum has zero amplitude above and below the Nyquist wave numbers $\pm K_{s} / 2$, these aliasing errors will affect the accuracy of the wave-number component estimate. The beam wave-number spectrum extends from $-\infty$ to $+\infty$ since it has finite dimensions [Eqs. (A8) and (A10)]. Therefore aliasing occurs no matter how large $K_{s}$ is. However, it will be greatly reduced when the Nyquist wave number is above the main peak of all the modes present in the bandwidth of interest, i.e., $K_{s} / 2>p \pi / L_{x}$ or $N_{d}>p$, where $p$ is the index of the highest mode found in the response. Also, the use of nonregular sampling is of interest. In this case, the aliasing effects can be reduced by designing some appropriate spectral window associated with a particular sampling scheme. Further investigation of spatial sampling and aliasing aspects will be the topic of a later paper.

\section{SENSOR DESIGN}

The previous section discussed prediction of sound radiation using analytical expressions for the frequency response functions of the radiation filters. This part addresses the design of the digital filters used to model the frequency response functions in the sensing procedure. Since the earlier sections have shown the equivalence of a Rayleigh's integral and a wave-number-based approach, the following derivations will only consider sensors based on the wavenumber information. The transfer functions derived before are first modified in order to optimize the number and length of the FIR filters. Two filter design methods in the frequency and time domains, respectively, are then de- 
scribed. The first design method is model-based; it uses the analytical transfer functions in the frequency domain. The second design method allows the use of time-domain experimental data measured on the structural acoustic system.

\section{A. Modified radiation transfer functions}

The choice of the time delay $\Delta \tau$ introduced in Sec. I B 2 is first discussed. The case of a 1-D structure $\left(k_{y}=0\right)$ is considered for simplicity. The structure is of finite width and its response is assumed to be constant in the $y$ direction (see Fig. 2).

The discrete wave-number component corresponding to the direction $\theta$ is written as the summation of the structural acceleration measured at a number of points $\left(x_{i}, y=0\right)$ equally spaced and multiplied by the radiation transfer functions $\bar{H}_{i}(\omega)\left(i=1,2, \ldots, N_{d}\right)$,

$$
\tilde{\ddot{W}}_{d}\left(k_{x}\right)=\sum_{i=1}^{N_{d}} \bar{H}_{i}(\omega) \ddot{W}\left(x_{i}\right) \text {. }
$$

Using a unit magnitude factor and a positive time delay $\Delta \tau$ to ensure causality in Eq. (24), the modified frequency response functions for the radiation filters are written as

$$
\bar{H}_{i}(\omega)=\exp \left(j \omega \bar{\tau}_{i}\right), \quad \bar{\tau}_{i}=\tau_{i}-\Delta \tau \leqslant 0 \quad\left(i=1,2, \ldots, N_{d}\right) .
$$

The number of coefficients of the $i$ th FIR filter should be at least greater than the time delay $\bar{\tau}_{i}$ divided by the sampling period $T_{s}=1 / F_{s}$. Therefore, it is desirable to make $\left|\bar{\tau}_{i}\right|$ as small as possible. An optimal value for $\Delta \tau$ is $\Delta \tau=\max \left\{\tau_{i}\right\}$ where $i=1,2, \ldots, N_{d}$. For this particular value of $\Delta \tau$, one of the delays $\bar{\tau}_{i}$ becomes zero while the others are minimum. As a first result, the number of coefficients of the FIR filters is optimized since the set of delays is made minimum. Second, the transfer function corresponding to the largest $\tau_{i}$ becomes equal to unity (zero time delay). Thus, the number of filters used in the sensor is reduced by one. In other words, the optimal $\Delta \tau$ results in a smaller computational effort. A rule of thumb to determine the number of coefficients $N_{i}$ can be expressed as

$$
N_{i}=R\left(\bar{\tau}_{i} / T_{s}\right)+\epsilon,
$$

where $R(x)$ rounds up the real $x$ (next integer to $x$ ), $T_{s}$ is the sampling period, and $\epsilon$ is an integer between 0 and 2 .

\section{B. Frequency domain design}

In the frequency domain, the filter coefficients are obtained by minimizing, in the least-square sense, the error between the desired and the modeled frequency response functions over the frequency range of interest. The desired frequency response is generated from Eq. (30). The modeled frequency response $H_{i}^{m}(\omega)$ can be found from the filter finite impulse response $\left\{U_{l}^{i}, l=0,1, \ldots, N_{i}-1\right\}$ as follows:

$$
H_{i}^{m}(\omega)=\sum_{l=0}^{N_{i}-1} U_{l}^{i} e^{-j \omega l T_{s}}, \quad i=1,2, \ldots, N_{d} .
$$

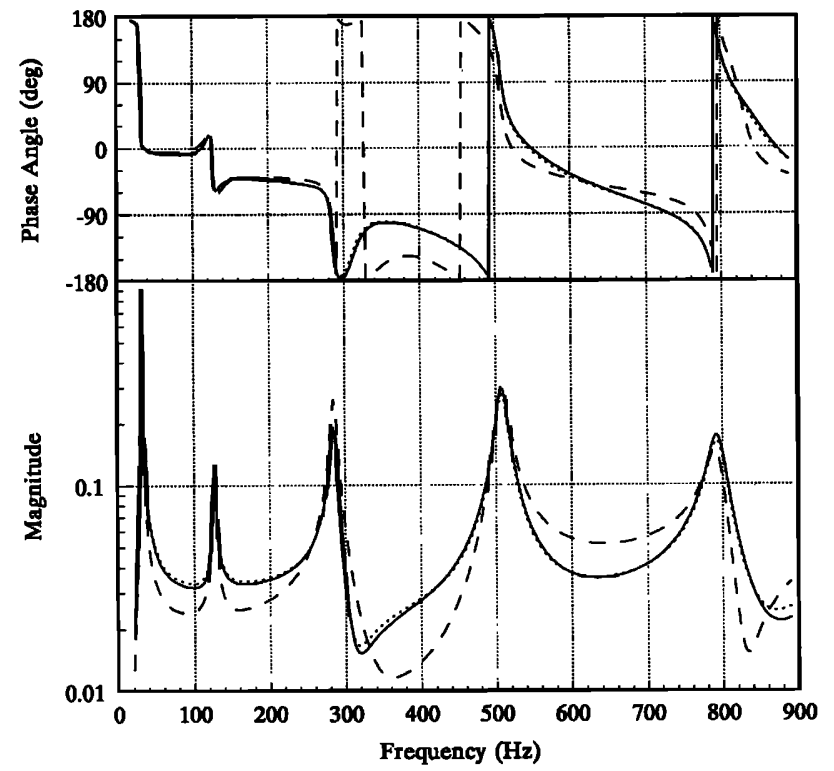

FIG. 5. Analytical and modeled sensor output: (a) — analytical discrete representation; (b) ----, (c)........, modeled discrete representations with $\epsilon=0$ and $\epsilon=1$, respectively.

Most of the existing frequency domain design algorithms allow the use of a weighting function such that the minimization be only effective over the frequency range of interest. Minimizing over the entire frequency range $[0, F, 2]$ would reduce the accuracy of the modeled frequency response function. The algorithm implemented in the Matlab function invfreqz (software by Math Works ${ }^{11}$ ) is used here. $^{12}$

To illustrate the design procedure, the discrete wavenumber component of the beam described earlier is now modeled by an array of filters. The sampling frequency is $F_{s}=3000 \mathrm{~Hz}$ and the design frequency range extends from 0 to $850 \mathrm{~Hz}$ including the first five bending modes. In this example, $N_{d}=4$ point sensors, i.e., four structural measurement points, are implemented to evaluate the wavenumber component corresponding to radiation in the direction $\theta=75^{\circ}$. Returning to Fig. 4 , this case corresponds to the curve (c). The optimal value for $\Delta \tau$ is determined from the time delays $\tau_{i}(i=1,2,3,4)$ as explained in the previous section. This choice implies that only three filters need to be designed, one transfer function being equal to unity. Using the rule of thumb given in Eq. (31) with $\epsilon=0$, the FIR filters have three, two, and one coefficient(s), respectively. A second case uses $\epsilon=1$, i.e., four, three, and two coefficients, respectively, are then determined. The sensor output spectrum is computed from the modeled frequency response functions of Eq. (32) and compared to the analytical discrete wave-number component. The results are presented in Fig. 5. The case $\epsilon=0$ (dashed line) gives a relatively good approximation at the resonant frequencies while some error is noticed off-resonance. Very small error is obtained with $\epsilon=1$ (dotted line). Note that since the delays $\tau_{i}$ are replaced by $\bar{\tau}_{i}=\tau_{i}-\Delta \tau$, the phase of the analytical and modeled wave-number components in 


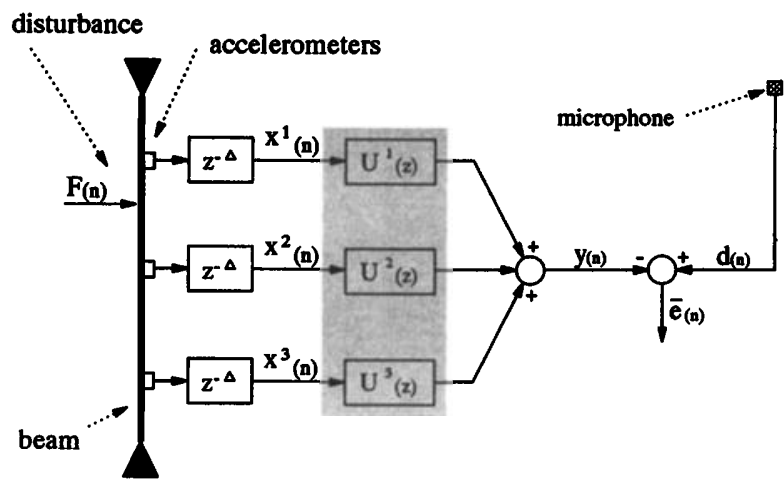

FIG. 6. Time domain sensor design applied to the simply supported beam.

Fig. 5 is different from the phase shown in Fig. 4 by a linear phase factor $\exp (-j \omega \Delta \tau / c)$.

\section{Time domain design}

The time domain design uses adaptive filter theory. ${ }^{9}$ It involves the measurements of the time varying structural response at each location $\left(x_{n_{x}}, y_{n_{y}}\right) \quad\left(n_{x}=1,2, \ldots, N_{x}\right.$, $n_{y}=1,2, \ldots, N_{y}$ ) along with the radiated far-field pressure in a prescribed direction. The filter coefficients can be found either on-line using the time domain LMS algorithm or off-line by computing the optimum least-square solution. In this method, the sensor includes the dynamics of the real system along with the response of the measuring instrumentation. This property is an advantage over the first design method, which uses analytical expressions and does not model the dynamics of the instrumentation. However, the time domain design depends on the excitation characteristics, i.e., the filter impulse responses represent the optimal solution over a given bandwidth for a particular type and location of excitation. For example, a sensor based on a particular disturbance input force may be inaccurate when the structure is controlled by the pair of moments induced by a piezoelectric actuator at a different location. However, these variations can be considered small enough compared to the degree of precision required for the sensor.

It has been shown previously that the time-varying wave-number component is proportional to the timeshifted pressure $p(\mathbf{r}, t+r / c)$. Thus, the desired sensor output $\bar{e}(t)$ can be expressed in function of the measured acoustic pressure as

$$
\bar{e}(t)=p[\mathbf{r}, t-\Delta \tau+(r / c)],
$$

where $\Delta \tau \geqslant 0$ accounts for causality. In addition to the causality requirement in Eq. (30), $\Delta \tau$ is required to satisfy $(r / c-\Delta \tau) / T_{s}=\Delta$, where $\Delta$ is an positive integer. The desired sensor output can then be expressed in the discrete time domain as $\bar{e}(n)=p(n+\Delta)$. Figure 6 shows the block diagram of the time domain sensor design in the case of a 1-D simply supported beam. It should be noted that the delay $z^{-\Delta}$ in the sensor path makes the system causal and equivalent to the time shift $z^{+\Delta}$ in the pressure path since $p(n)=\bar{e}(n-\Delta)=d(n)$. The error to be minimized is the difference between the measured pressure and the sensor output: $e(n)=d(n)-\bar{e}(n)$. The acceleration at time $t_{n-\Delta}$ measured at point $x_{i}$ is denoted $x^{i}(n)$. The error signal $e(n)$ can be expressed in matrix form by the following general formula:

$$
e(n)=d(n)-\mathbf{X}^{T}(n) \mathbf{U}=d(n)-\mathbf{U}^{T} \mathbf{X}(n) .
$$

The column vector $\mathrm{U}$ contains the $N_{i}$ coefficients of the filters $\underline{U}^{i}\left(i=1, \ldots, N_{d}\right)$,

$$
\mathbf{U}=\left\{\begin{array}{c}
\left\{\underline{U}^{1}\right\} \\
\vdots \\
\left\{\underline{U}^{i}\right\} \\
\vdots \\
\left\{\underline{U}^{N_{d}}\right\}
\end{array}\right\}, \quad \text { where } \underline{U}^{i}=\left\{\begin{array}{c}
U_{0}^{i} \\
\vdots \\
U_{N_{i}-1}^{i}
\end{array}\right\}
$$

The column vector $\mathbf{X}(n)$ is defined as

$$
\mathbf{X}(n)=\left\{\begin{array}{c}
\left\{\underline{x}^{1}(n)\right\} \\
\vdots \\
\left\{\underline{x}^{i}(n)\right\} \\
\vdots \\
\left\{\underline{x}^{N_{d}}(n)\right\}
\end{array}\right\}, \quad \text { where } \underline{x}^{i}(n)=\left\{\begin{array}{c}
x^{i}(n) \\
x^{i}(n-1) \\
\vdots \\
x^{i}\left(n-N_{i}+1\right)
\end{array}\right\}
$$

Here, $\mathbf{X}(n)$ and $\mathrm{U}$ both contain $K=\Sigma_{i=1}^{N_{d}} N_{i}$ elements $X_{k}(n)$ and $U_{k}(k=1, \ldots, K)$, respectively. Equation (34) can be rewritten in closed form as

$$
\begin{aligned}
e(n) & =d(n)-\sum_{i=1}^{N_{d}}\left\{\sum_{j=0}^{N_{i}-1} U_{j}^{i} x^{i}(n-j)\right\} \\
& =d(n)-\sum_{k=1}^{K} U_{k} X_{k}(n) .
\end{aligned}
$$

The cost function to be minimized is defined as the meansquare value of the error signal,

$$
\begin{aligned}
J\left(U_{k}\right)= & E\left\{e^{2}(n)\right\} \\
= & E\left\{d^{2}(n)\right\}-2 E\left\{d(n) \mathbf{X}^{T}(n)\right\} \mathbf{U} \\
& +\mathbf{U}^{T} E\left\{\mathbf{X}(n) \mathbf{X}^{T}(n)\right\} \mathbf{U} .
\end{aligned}
$$

The above expression is a quadratic function of the filter coefficients $U_{k}$. Thus there is only one optimal solution. Differentiating $J\left(U_{k}\right)$ with respect to $U_{k}$ in Eq. (38) produces

$$
\frac{\partial J}{\partial U_{k}}\left(U_{k}\right)=2 E\left\{e(n) \frac{\partial e(n)}{\partial U_{k}}\right\}=-2 E\left\{e(n) X_{k}(n)\right\},
$$

where $X_{k}(n)$ denotes the $k$ th element of $\mathbf{X}(n)$. The optimum solution can be obtained by substituting Eq. (37) in Eq. (39) and setting the partial derivatives with respect to $U_{k}$ equal to 0 for $k=1,2, \ldots, K$. These $K$ linear equations are rearranged in matrix form as

$$
\mathbf{R}_{x x} \mathbf{U}=\mathbf{R}_{d x} .
$$

Here, $\mathbf{R}_{x x}$ denotes the autocorrelation matrix of the input signals contained in the vector $\mathbf{X}(n)$ and $\mathbf{R}_{d x}$, the crosscorrelation matrix between the input signals and the desired output signal $d(n)$ : 


$$
\begin{gathered}
\mathbf{R}_{x x}=\left[\begin{array}{c}
E\left\{X_{1}(n) \mathbf{X}^{T}(n)\right\} \\
E\left\{X_{2}(n) \mathbf{X}^{T}(n)\right\} \\
\vdots \\
E\left\{X_{K}(n) \mathbf{X}^{T}(n)\right\}
\end{array}\right], \\
\mathbf{R}_{d x}=\left[\begin{array}{c}
E\left\{d(n) X_{1}(n)\right\} \\
E\left\{d(n) X_{2}(n)\right\} \\
\vdots \\
E\left\{d(n) X_{K}(n)\right\}
\end{array}\right] .
\end{gathered}
$$

Alternatively, the LMS algorithm ${ }^{9}$ can be used to adapt the coefficients $U_{k}(k=1,2, \ldots, K)$ in order to minimize the cost function $J\left(U_{k}\right)$ using the steepest descent method,

$$
U_{k}(n+1)=U_{k}(n)-\mu \frac{\partial J}{\partial U_{k}}\left(U_{k}\right),
$$

where $\mu$ controls the stability and rate of convergence. The time domain LMS algorithm uses an instantaneous gradient approximation yielding the following update equation:

$$
U_{k}(n+1)=U_{k}(n)+2 \mu e(n) X_{k}(n) \quad(k=1,2, \ldots, K) .
$$

\section{CONCLUSIONS}

A new sensing technique using point structural sensors has been developed for the prediction of far-field pressure or structural wave-number information in the case of vibrating finite rectangular radiators. The approach is based on the discretization of the Helmoltz integral over the radiating surface. By choosing an appropriate Green's function, the term related to the surface pressure vanishes and the radiated pressure becomes only a function of the radiating surface acceleration. The practical implementation uses accelerometers to measure the structural response and FIR filters to model the transfer functions associated with each elemental area. The summation of the filter outputs gives a good estimate of the far-field pressure radiated in a prescribed direction or its equivalent wave-number expression. When applied to feedforward control approaches, the filter array is optimized to considerably reduce the computational load thus making the technique easy to implement on real structures.

As opposed to the use of distributive sensors that filter the efficient radiating modes in the spatial domain using shaped PVDF films, the present method filters the structural information in the time domain using digital filters. Filters being easier to design and more selective than PVDF films, this real time structural acoustic sensor is believed to yield better control performances in a prescribed direction. In addition, unlike PVDF films that are shaped for a single modal response, the described sensor gives radiation information over a broadband frequency range.

The present approach is only valid for the case of planar radiators. In the case of more complex geometry, the Rayleigh's integral cannot be used and a formulation involving another type of Green's function needs to be derived or the structural diffraction terms accounted for.
Further investigation will extend the technique to those cases and experimentally demonstrate its use on real structures.

\section{ACKNOWLEDGMENTS}

The authors gratefully acknowledge the support of this work by the Office of Naval Research under Grant No. ONR-N00014-92-j-1170.

\section{APPENDIX}

Analytical expressions for the beam response, its continuous and discrete wave-number transforms are derived. The beam shown in Fig. 2 is simply supported with length $L_{x}$ and width $L_{y}$. An harmonic point force $f(t)=F e^{j \omega t}$ is applied at location $\left(x=x_{0}, y=0\right)$. The beam response is assumed to have constant amplitude of motion in the $y$ direction and the radiated acoustic field is investigated in the $x-z$ plane $(\phi=0)$. The surrounding media is air; it is assumed to have negligible influence on the in vacuo structural response.

\section{Structural response}

The beam out-of-plane displacement complex amplitude $W(x)$ can be expressed as an infinite summation over each modal response

$$
W(x)=\sum_{p=1}^{+\infty} q_{p}(\omega) \psi_{p}(x), \quad-\frac{L_{x}}{2} \leqslant x \leqslant \frac{L_{x}}{2},
$$

where $q_{p}(\omega)$ is the $p$ th modal displacement and $\psi_{p}(x)$ is the $p$ th eigenfunction. Taking the origin of the coordinate system at the center of the beam, the eigenfunctions corresponding to the simply supported boundary condition are

$$
\psi_{p}(x)=(2 / m)^{1 / 2} \sin \left[\gamma_{p}\left(x+L_{x} / 2\right)\right],
$$

where $\gamma_{p}=p \pi / L_{x}$. The factor $(2 / m)^{1 / 2}$ results from the normalization chosen for the eigenfunctions, such that they are orthonormal with respect to the mass $m$ of the beam. The modal displacement $q_{p}(\omega)$ is given by

$$
q_{p}(\omega)=H_{p}(\omega) \psi_{p}\left(x_{0}\right) F=\frac{1}{\omega_{p}^{2}-\omega^{2}+2 j \eta_{p} \omega_{p} \omega} \psi_{p}\left(x_{0}\right) F,
$$

where $H_{p}(\omega)$ is the $p$ th modal frequency response function. Here, $\omega_{p}$ and $\eta_{p}$ are the $p$ th natural frequency and modal damping ratio, respectively. The analytical natural frequencies are defined as

$$
\omega_{p}^{2}=\gamma_{p}^{4} \frac{E I}{\rho_{s} S_{s}},
$$

where $\rho_{s}$ is the beam density, $S_{s}$ the beam section, and $E I$ is the Young's modulus bending inertia product. The acceleration response is obtained by taking the second partial derivative with respect to time of the displacement response,

$$
\ddot{w}(x, t)=\ddot{W}(x) e^{j \omega t}=-\omega^{2} W(x) e^{j \omega t} .
$$


The beam response is now sampled with $N_{d}$ points at locations $x_{i}=-L_{x} / 2+\Delta x / 2(2 i-1), i=1,2, \ldots, N_{d}$, where $\Delta x=L_{x} / N_{d}$ is the sampling period. The discrete acceleration response can be expressed using Dirac distributions as

$$
\ddot{W}_{d}(x)=\sum_{i=1}^{N_{d}} \ddot{W}(x) \Delta x \delta\left(x-x_{i}\right) .
$$

This representation will be useful when deriving an alternative expression for the discrete wave-number transform.

\section{Wave-number transform}

Substituting $k_{y}=0$ in Eq. (15) yields the following single integral for the one-dimensional acceleration wavenumber transform:

$$
\tilde{\ddot{W}}\left(k_{x}\right)=L_{y} \int_{-L_{x} / 2}^{L_{x} / 2} \ddot{W}(x) e^{j k_{x} x} d x .
$$

Equation (A7) can be rewritten in terms of the modal acoustic influence functions as

$$
\tilde{\ddot{W}}\left(k_{x}\right)=-\omega^{2} \sum_{p=1}^{+\infty} q_{p}(\omega) \xi_{p}\left(k_{x}\right),
$$

where

$$
\xi_{p}\left(k_{x}\right)=L_{y} \int_{-L_{x} / 2}^{L_{x} / 2} \psi_{p}(x) e^{j k_{x} x} d x .
$$

Here, $\xi_{p}\left(k_{x}\right)$ is defined as the wave-number transform of the $p$ th eigenfunction. Hence, it can also be referred to as the $p$ th modal wave-number component. Substituting Eq. (A2) in Eq. (A9) yields

$$
\xi_{p}\left(k_{x}\right)=L_{y}\left(\frac{2}{m}\right)^{1 / 2}\left[\begin{array}{c}
\cos \left(k_{x} L_{x} / 2\right) \\
-j \sin \left(k_{x} L_{x} / 2\right)
\end{array}\right] \frac{2 \gamma_{p}}{\left(\gamma_{p}^{2}-k_{x}^{2}\right)},
$$

where $\cos \left(k_{x} L_{x} / 2\right)$ is used when $p$ is odd and $\sin \left(k_{x} L_{x} / 2\right)$ when $p$ is even. As seen in Eq. (A10), $\xi_{p}\left(k_{x}\right)$ is either purely real (odd mode number) or purely imaginary (even mode number). Its zeros are expressed as

$k_{x}=\left\{\begin{aligned}(2 n+1) \gamma_{p}, & \text { for } p \text { odd, } n \text { integer } \\ & \text { and } 2 n+1 \neq \pm p, \\ 2 n \gamma_{p}, & \text { for } p \text { even, } n \text { integer, } \\ & \text { and } 2 n \neq \pm p .\end{aligned}\right.$

\section{Discrete wave-number transform} erator,

Let $\mathscr{K}\{g(x)\}$ denote the wave-number transform op-

$$
\mathscr{K}\{g(x)\}=\int_{-\infty}^{+\infty} g(x) e^{+j k_{x} x} d x .
$$

Using the discrete representation in Eq. (A6), the discrete wave-number transform is expressed as

$$
\begin{aligned}
\tilde{\ddot{W}}_{d}\left(k_{x}\right) & =L_{y} \mathscr{K}\left\{\ddot{W}_{d}(x)\right\} \\
& =L_{y} \mathscr{K}\left\{\ddot{W}(x) \Delta x \sum_{i=1}^{N_{d}} \delta\left(x-x_{i}\right)\right\} .
\end{aligned}
$$

An important property of Fourier transforms yields

$$
\begin{aligned}
\tilde{\ddot{W}}_{d}\left(k_{x}\right) & =L_{y} \mathscr{K}\{\ddot{W}(x)\} * \mathscr{K}\left[\Delta x \sum_{i=1}^{N_{d}} \delta\left(x-x_{i}\right)\right\} \\
& =\tilde{\ddot{W}}\left(k_{x}\right) * \delta_{N}\left(k_{x}\right),
\end{aligned}
$$

where $*$ denotes the convolution product and $\delta_{N}\left(k_{x}\right)$ is a spectral window given by

$$
\delta_{N}\left(k_{x}\right)=\Delta x \sum_{i=1}^{N_{d}} e^{j k_{x} x_{i}}
$$

Substituting $x_{i}$ in terms of $L_{x}, \Delta x$, and $i$, the above expression can be rewritten as

$$
\delta_{N}\left(k_{x}\right)=\Delta x \frac{\sin \left(k_{x} N_{d} \Delta x / 2\right)}{\sin \left(k_{x} \Delta x / 2\right)} e^{j k_{x}\left(-L_{x}+N_{d} \Delta x\right) / 2} .
$$

The magnitude of $\delta_{N}\left(k_{x}\right)$ takes its maximum value at an infinite set of wave numbers $k_{x}=n K_{s}$, where $K_{s}=2 \pi / \Delta x$ is the spatial sampling frequency and $n$ is an integer. It is symmetric and periodic with period $K_{s}$, i.e., $\left|\delta_{N}\left(-k_{x}\right)\right|=\left|\delta_{N}\left(k_{x}\right)\right|$ and $\left|\delta_{N}\left(k_{x}\right)\right|=\left|\delta_{N}\left(k_{x}+n K_{s}\right)\right|$. In brief, $\left|\delta_{N}\left(k_{x}\right)\right|$ is approximately like an infinite row of sinc functions spaced $K_{s}$ apart.

${ }^{1}$ J. D. Maynard, E. G. Williams, and Y. Lee, "Nearfield acoustic holography: I. Theory of generalized holography and the development of NAH," J. Acoust. Soc. Am. 78, 1385-1413 (1985).

${ }^{2}$ S. Amini, C. Ke, and P. J. Harris, "Iterative solution of boundary element equations for the exterior Helmoltz problem," J. Vib. Acoust. 112, 257-262 (1990)

${ }^{3}$ C. R. Fuller, C. A. Rogers, and H. H. Robertshaw, "Control of sound radiation with active/adaptive structures," J. Sound Vib. 157(1), 19-39 (1992).

${ }^{4}$ R. L. Clark and C. R. Fuller, "Modal sensing of efficient radiators with PVDF distributed sensors in active structural acoustic approaches," J. Acoust. Soc. Am. 91, 3321-3329 (1990).

${ }^{5}$ W. T. Baumann, W. R. Saunders, and H. H. Robertshaw, "Active suppression of acoustic radiation from impulsively excited structures," J. Acoust. Soc. Am. 90, 3202-3208 (1991).

${ }^{6}$ M. C. Junger and D. Feit, Sound, Structures and Their Interaction (MIT, Boston, 1986).

${ }^{7}$ F. J. Fahy, Sound and Structural Vibration (Academic, London, 1985).

${ }^{8}$ C. R. Fuller and R. A. Burdisso, "A wavenumber domain approach to the active control of structure-borne sound," J. Sound Vib. 148(2), 355-360 (1991).

${ }^{9}$ B. J. Widrow and S. D. Stearns, Adaptive Signal Processing (PrenticeHall, Englewood Cliffs, NJ, 1986).

${ }^{10}$ R. L. Clark and C. R. Fuller, "A model reference approach for implementing active structural acoustic control," J. Acoust. Soc. Am. 92 , 1534-1544 (1992).

${ }^{11}$ PC-Matlab for MS-DOS Computers (The MathWorks, Inc., South Natick, MA, 1989).

${ }^{12}$ J. O. Smith, "Techniques for Digital Filter Design and System Identification, with Application to the Violin," Ph.D. dissertation, Stanford University (1983). 